\title{
SITUACIÓN ACTUAL DE LA LENGUA FRANCESA EN LA ENSEÑANZA PRIMARIA Y SECUNDARIA DENTRO DEL TERRITORIO DEL MEC
}

Rosa M." Calvet LoRA

UNED

La dramática situación que atraviesan los estudios de Filología Francesa en la Universidad española tiene mucho que ver con la falta de interés hacia esa lengua en la enseñanza secundaria y en la primaria. Por esta razón me parece importante presentar algunos datos que reflejan la situación actual de los estudios de francés tanto en la enseñanza primaria como en la secundaria dentro del territorio del MEC, que pueden ayudar a conocer la magnitud de un problema que nos afecta directamente.

Estos datos que presento han sido elaborados a partir del recuento del número de alumnos matriculados en las diversas opciones de lenguas extranjeras que se ofrecen en nuestro país. He comenzado el estudio desde el curso 198990. Son datos aún provisionales, ya que falta una comprobación definitiva por parte del departamento de procesamiento de datos del Ministerio, aunque no parece que las modificaciones que puedan producirse den lugar a cambios espectaculares en el asunto que nos ocupa. Del curso actual, 92/93 sólo puedo ofrecer datos porcentuales, ya que no he tenido aún acceso al desglose de los mismos.

La presentación de los datos se organiza de la siguiente manera:

En todas las tablas se presentan por una parte los diferentes idiomas que se ofrecen con la distinción entre enseñanza pública y enseñanza privada. Dentro 
de la enseñanza pública existen aquellos centros públicos directamente gestionados por el MEC y otros centros públicos. Dentro de la enseñanza privada existe la diferenciación entre centros gestionados por la Iglesia católica y resto de centros privados. He englobado en dos únicos apartados; centros públicos y centros privados para evitar atomizaciones innecesarias, y en aras de, en mi opinión, una mayor claridad.

En primer lugar, se presentan los alumnos de los idiomas obligatorios dentro del actual sistema educativo que cursan estudios de B.U.P. y C.O.U., tanto divididos por cursos como el total global. Seguidamente, se presentan el número de alumnos de los mismos cursos de BUP y COU que cursan idioma optativo en aquellos casos en que esta opción es posible.

Posteriormente se presentan los mismos datos con los alumnos que cursan formación profesional, para finalizar con la relación de los alumnos de E.G.B., que dentro del plan actual, como sabemos, comienzan a cursar idioma obligatorio a partir de $60^{\circ}$ En el caso de estos alumnos he incluído además el dato de «otras lenguas españolas» que me ha parecido significativo.

Detrás de cada grupo de datos en cifras, presento los mismos datos en porcentajes, pues me parece el modo más fácil y evidente de contrastar las diferentes cantidades. Los porcentajes parciales se remiten siempre al total global situado al final de cada línea, por ejemplo: en el curso 89/90 en $1 .^{\circ}$ de BUP el $87 \%$ de alumnos que cursan inglés en la enseñanza pública está referido al total de alumnos que cursan idiomas en la enseñanza pública, y así, con todos.

En último lugar presento los datos de los alumnos inscritos dentro del plan experimental de las enseñanzas medias.

Comencemos el análisis de los datos: analizaremos primero la posición del francés respecto del inglés cuando se trata de la opción de lengua obligatoria. De esta relación, sacaremos algunas consideraciones puntuales:

- La primera de ellas, evidentemente, es la que todos sabemos: la aplastante mayoría de alumnos que eligen inglés como lengua extranjera, así como la escasa incidencia de los otros idiomas en la posible elección, decantándose el francés casi como única alternativa.

- En segundo lugar, constatamos que el porcentaje de alumnos que cursan inglés es mayor en general en los centros privados que en los públicos, mientras que disminuye consecuentemente aún más el porcentaje de estudiantes de francés en los centros privados. Sabemos que son numerosos los centros privados que prescinden directamente de ofrecer el francés como opción, mientras que ésta se mantiene en los centros públicos. 
- En tercer lugar, es también general el hecho de que el porcentaje de alumnos de francés aumenta en relación directa a la progresión en el curso, es decir, es mayor el número en COU que en $10^{\circ}$ de BUP, por ejemplo, o en el segundo grado de FP que en el primero, lo que evidencia que las nuevas generaciones que van accediendo a los estudios secundarios optarán cada vez menos por el francés.

- Cuarta consideración: los porcentajes decrecen paulatinamente, en razón de una media de dos puntos por curso en FP y de más de tres en BUP y COU: de un total de $17,4 \%$ en el curso $89 / 90$ hemos pasado a un $8,2 \%$ en el curso $92 / 93$, perdiéndose 9.2 puntos.

En E.G.B. pasamos del curso $90 / 91$ al actual, del $9,8 \%$ al $5 \%$ con lo que se pierden 4.8 puntos.

En FP pasamos del $89 / 90$ con $15,1 \%$ al $10 / \%$ del curso $92-93$, con una pérdida de 5.1 puntos.

Es decir, parece que aún no se ha tocado fondo, ni se ha conseguido una estabilización, sino que el proceso descendente continúa.

Pasemos ahora a la consideración del francés como lengua optativa. En primer lugar dentro del plan actual.

Sabemos las dificultades que existen actualmente en los centros para poner en práctica los programas de una lengua extranjera optativa: complicación en los horarios, una dificultad añadida al currículo de los alumnos, etc. Sin embargo, podemos observar un panorama bastante alentador:

- En primer lugar la gran mayoría de los alumnos que han elegido cursar una lengua extranjera optativa se han decantado por el francés.

- En segundo lugar se da con el francés la tendencia contraria a lo que sucedía con la lengua obligatoria: el porcentaje aumenta en los primeros cursos, es decir que los nuevos alumnos son cada vez más receptivos a la importancia de la segunda lengua.

- En tercer lugar el creciente aumento en los porcentajes. Si exceptuamos un leve retroceso en el curso 91-92, pasamos del 87,7\% en BUP y COU en el $89 / 90$ al $96,7 \%$ en el curso actual, con un aumento de 9 puntos.

- Hay que mencionar que el alemán se perfila como la tercera opción de lengua extranjera y que su número de alumnos crece. Según he podido constatar en un estudio sólo del curso 1990-91, es Cataluña la Comunidad Autónoma que tiene más alumnos de alemán. 
Respecto a la E.G.B. la tendencia es la misma, aunque no existe tanta diferencia entre centros públicos y privados en lo que al francés se refiere.

Examinemos a continuación el plan experimental de las enseñanzas medias, es decir, de aquellos centros que han puesto en marcha la Reforma y que hasta este curso suponen unos 300 . Es de estos datos de Jos que más claramente podemos inferir el futuro del francés en la secundaria.

Aunque es de todos conocida, recordaré brevemente el nuevo esquema de la enseñanza secundaria obligatoria, que se organiza en dos ciclos de dos cursos cada uno: el primero va de los 12 años a los 14 y el $2^{\circ}$ de los 14 a los 16 . Posteriormente, el bachillerato constará de dos cursos.

En el cuarto año de la etapa los alumnos han de elegir dos entre las cuatro áreas siguientes, según se establece en el currículo de la Educación Secundaria Obligatoria (Real Decreto 1345/1991, de 6 de septiembre, B.O.E. n. 220 , de 13-IX-1991): Ciencias de la Naturaleza, Educación Plástica y Visual, Música y Tecnología. Además de esta posibilidad, el currículo comprenderá materias optativas (artículo $7 .^{\circ}, 1$ ). La oferta de materias optativas de los centros, en cada curso y a lo largo de la etapa, deberá ser suficientemente diversa y equilibrada (art. 7., 2 ). Este mismo artículo determina aquellas materias optativas que son oferta obligada para los centros: entre las materias optativas se incluirán en todo caso una segunda lengua extranjera durante toda la etapa, una materia de iniciación profesional en el segundo ciclo, y cultura clásica al menos en un curso del segundo ciclo.

El número de materias que han de cursar los alumnos será de una en tercer curso y dos en cuarto curso, siempre que la organización temporal de las materias elegidas sea de curso completo. Excepcionalmente, este número podrá modificarse con organizaciones temporales distintas, trimestrales o cuatrimestrales, siempre que la suma de los tiempos coincida con el horario total dedicado al espacio de optatividad en cada curso (dos horas en tercer curso y seis en cuarto curso).

Así pues el francés se va obligado a competir ahora en el espacio de la optatividad con materias como: Taller de Artesanía, Taller de Astronomía, Los Procesos de Comunicación, Imagen y Expresión, Taller de Teatro, Canto Coral, Taller de Matemáticas, Expresión Corporal, Transición a la Vida Adulta y Activa. La única ventaja es que es materia optativa de oferta obligatoria para los centros.

El número de horas que está previsto en los diseños curriculares asignar a la primera lengua extranjera y a la segunda lengua optativa son los siguientes: 


\section{- Lengua extranjera obligatoria}

Enseñanza primaria: la lengua extranjera se introduce en el segundo ciclo, es decir a partir de los ocho años de edad, correspondiendo al segundo ciclo 170 horas y al tercer ciclo 170 horas. Dentro de la enseñanza secundaria obligatoria corresponden 210 horas al primer ciclo ( $10^{\circ}$ y $2 .^{\circ}$ cursos) y 240 horas al $2 .^{\circ}$ ciclo (tercero y cuarto cursos).

En cuanto al bachillerato se preven 3 horas semanales tanto en el primer curso como en el segundo curso.

\section{- Segunda lengua optativa}

Se le dedicarán dos horas semanales en el tercer curso de enseñanza secundaria obligatoria y 3 horas semanales en el cuarto curso.

Dentro del bachillerato, la segunda lengua optativa, tiene asignadas más horas que la primera lengua obligatoria, ya que se dedicarán 4 horas semanales tanto en primero como en segundo curso. La filosofía de esta distribución se basa en que una vez que los alumnos disponen de las estrategias de aprendizaje de una primera lengua extranjera así como las de la lengua materna, a pesar de la introducción posterior de la segunda lengua, el resultado en cuanto a dominio puede llegar a ser muy similar.

Los datos que nos ofrece el cuadro de la REM son similares a los del plan actual en cuanto al idioma obligatorio, es más los porcentajes por años son casi idénticos, lo que indica que el descenso forma parte del mismo fenómeno.

Debemos tener en cuenta una tendencia a la baja que se aprecia entre los dos cursos de los que disponemos datos en cuanto a la opcionalidad, y fijar la atención en el aumento de los porcentajes de las otras lenguas. Debemos potenciar y desarrollar la segunda lengua optativa y competir con las otras materias que se ofertan dentro del mismo bloque. Para ello es preciso concienciar a la sociedad de la importancia que tiene el conocimiento de lenguas y no el de una sola lengua extranjera, partiendo de realidades concretas, como por ejemplo del hecho de que para trabajar en cualquier organismo de la CEE se precisan cuando menos dos lenguas extranjeras, además de la materna.

En Europa la enseñanza de dos lenguas extranjeras es práctica generalizada. En España se ha potenciado únicamente el estudio de una de ellas, por lo que naturalmente, el francés perdió hace muchos años la batalla. 
ANEXo 1

\begin{tabular}{|c|c|c|c|c|c|c|c|c|c|c|c|c|c|c|c|c|c|c|}
\hline & & & & MEC & ALU & ANO & DE B & U.P y & .0 .0 & que $c$ & ursan i & liom & obligs & torio & & & & \\
\hline \multirow{2}{*}{ C: 89150} & \multicolumn{3}{|c|}{ NGLES } & \multicolumn{3}{|c|}{ FRANCÉS } & \multicolumn{3}{|c|}{ ALEMÁN } & \multicolumn{3}{|c|}{ ITALANO } & \multicolumn{3}{|c|}{ OTROS } & \multicolumn{3}{|c|}{ TOTAL } \\
\hline & Piblico & Privado & Total & Público & Privado & Total & Público & Privado & Total & Público & Privado & Toul & Público & Privado & Total & Püblico & Privado & Total \\
\hline BUP I & 111303 & 54263 & 165566 & 15114 & 3088 & 18202 & 172 & 252 & 424 & 110 & 55 & 165 & 41 & 0 & 41 & 126740 & 57658 & 184398 \\
\hline BUP 2 & 92922 & 51544 & 144466 & 17737 & 3829 & 21566 & 153 & 228 & 381 & 83 & 46 & 129 & 11 & 0 & 11 & 110906 & 55647 & 166553 \\
\hline BUP 3 & 75074 & 43929 & 119003 & 19266 & 4189 & 23455 & 122 & 213 & 335 & 66 & 49 & 115 & 6 & 0 & 6 & 94534 & 48380 & 142914 \\
\hline $\mathrm{COU}$ & 66712 & 36758 & 103470 & 21007 & 4060 & 25067 & 109 & 190 & 299 & 55 & 37 & 92 & 13 & 0 & 13 & 87896 & 41045 & 128941 \\
\hline TOTAL & 346011 & 186494 & 532505 & 73124 & 15166 & 88290 & 556 & 883 & 1439 & 314 & 187 & $\mathbf{S O 1}$ & 71 & 0 & 71 & 420076 & 202730 & 622806 \\
\hline \multirow{2}{*}{$C: 9091$} & \multicolumn{3}{|c|}{ INGLES } & \multicolumn{3}{|c|}{ FRANCES } & \multicolumn{3}{|c|}{ ALEMÁN } & \multicolumn{3}{|c|}{ TTALANO } & \multicolumn{3}{|c|}{ OTROS } & \multicolumn{3}{|c|}{ TOTAL } \\
\hline & Público & Privado & Total & Público & Privado & Total & Público & Privado & Total & Público & Privado & Total & Público & Privado & Total & Público & Privedo & Total \\
\hline BUP I & 110109 & 53068 & 163177 & 10256 & 1853 & 12109 & 185 & 93 & 278 & & & 0 & & & 0 & 120550 & 55014 & 175564 \\
\hline BUP 2 & 95747 & 49951 & 145698 & 13106 & 2237 & 15343 & 145 & 114 & 259 & & & 0 & & & 0 & 108998 & 52302 & 161300 \\
\hline BUP 3 & 79136 & 45276 & 124412 & 14922 & 2776 & 17698 & 152 & 83 & 235 & & & 0 & & & 0 & 94210 & 48135 & 142345 \\
\hline $\mathrm{COU}$ & 70975 & 37683 & 108658 & 17029 & 2853 & 19882 & 105 & 50 & 155 & & & 0 & & & 0 & 88109 & 40586 & 128695 \\
\hline TOTAL & $355 \% 7$ & 185978 & 541945 & 55313 & 9719 & 65032 & 587 & 340 & 927 & 0 & 0 & 505 & 0 & 0 & 32 & 411867 & 196037 & 608441 \\
\hline \multirow{2}{*}{ C: $91 / 92$} & \multicolumn{3}{|c|}{ INGLES } & \multicolumn{3}{|c|}{ FRANCES } & \multicolumn{3}{|c|}{ ALEMÁN } & \multicolumn{3}{|c|}{ ITALANO } & \multicolumn{3}{|c|}{ OTROS } & \multicolumn{3}{|c|}{ TOTAL } \\
\hline & Püblico & Privado & Total & Público & Privado & Total & Público & Privado & Total & Público & Privado & Total & Píblico & Privado & Total & Público & Privado & Total \\
\hline BUP 1 & 105616 & 51799 & 157415 & 7255 & 1054 & 8309 & 240 & 89 & 329 & 68 & 56 & 124 & & & 0 & 11317 & 52998 & 166177 \\
\hline BUP 2 & 99452 & 50582 & 150034 & 10616 & 1398 & 12014 & 183 & 83 & 266 & 109 & 51 & 160 & & & 0 & 110360 & 52114 & 162474 \\
\hline BUP 3 & 85292 & 45462 & 130754 & 11881 & 1750 & 13631 & 137 & 96 & 233 & 92 & 35 & 127 & & & 0 & 9740 & 47343 & 144745 \\
\hline COU & 77325 & 3960 & 116985 & 14534 & $22 \%$ & 16830 & 123 & 57 & 180 & 61 & 39 & 100 & & & 0 & 9204 & 42052 & 134095 \\
\hline TOTAL & 367685 & 187503 & 555188 & 44286 & 6498 & 50784 & 683 & 325 & 1008 & 330 & 181 & 511 & 0 & 0 & 0 & 41298 & 194507 & 607491 \\
\hline
\end{tabular}




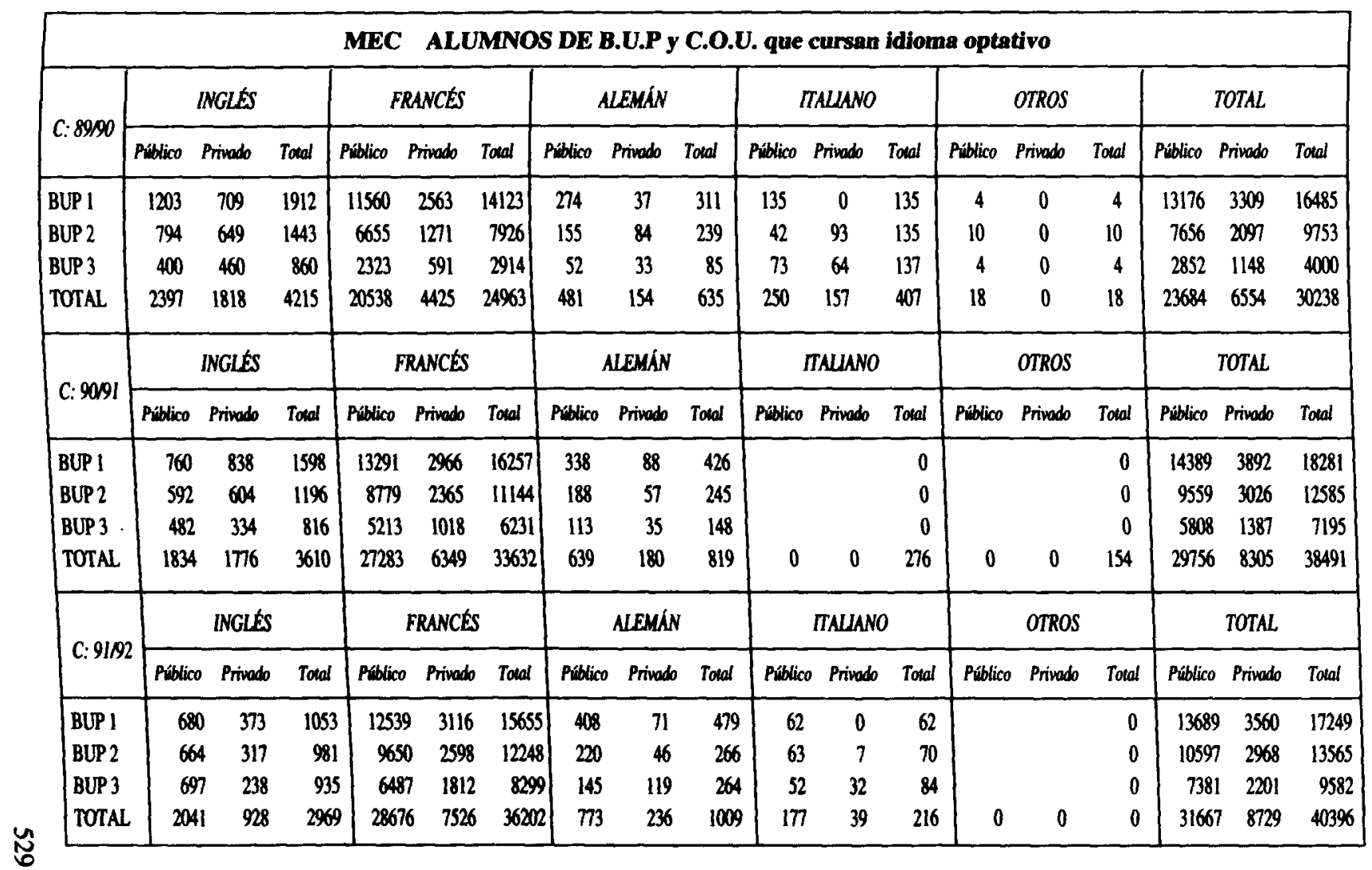


ANEXO 1.1

MEC ALUMNOS DE B.U.P y C.O.U. que cursan idioma obligatorio

\begin{tabular}{|c|c|c|c|c|c|c|c|c|c|c|c|c|c|c|c|c|c|}
\hline \multirow{2}{*}{$C: 89 / 90$} & \multicolumn{3}{|c|}{ INGLÉS } & \multicolumn{3}{|c|}{ FRANCÉS } & \multicolumn{3}{|c|}{ ALEMÁN } & \multicolumn{3}{|c|}{ ITALIANO } & \multicolumn{3}{|c|}{ OTROS } & \multicolumn{2}{|l|}{ TOTAL } \\
\hline & Público & Privado & Total & Público & Privedo & Total & Püblico & Privado & Total & Público & Privado & Total & Público & Privado & Total & Públice Privado & Total \\
\hline BUP 1 & $87.8 \%$ & $94.1 \%$ & $89.8 \%$ & $1.9 \%$ & $5.4 \%$ & $9.9 \%$ & $0.1 \%$ & $0.4 \%$ & $0.2 \%$ & $0.1 \%$ & $0.1 \%$ & $0.1 \%$ & $0.0 \%$ & 0.0 & $0.0 \%$ & $100.0 \% \quad 100.0 \%$ & $100.0 \%$ \\
\hline BUP2 & $83.8 \%$ & $92.6 \%$ & $86.7 \%$ & $16.0 \%$ & $6.9 \%$ & $12.9 \%$ & $0.1 \%$ & $0.4 \%$ & $0.2 \%$ & $0.1 \%$ & $0.1 \%$ & $0.1 \%$ & $0.0 \%$ & $0.0 \%$ & $0.0 \%$ & $100.0 \% \quad 100.0 \%$ & $100.0 \%$ \\
\hline BUP 3 & $79.4 \%$ & $90.8 \%$ & $83.3 \%$ & $20.4 \%$ & $8.7 \%$ & $16.4 \%$ & $0.1 \%$ & $0.4 \%$ & $0.2 \%$ & $0.1 \%$ & $0.1 \%$ & $0.1 \%$ & $0.0 \%$ & $0.0 \%$ & $0.0 \%$ & $100.0 \% \quad 100.0 \%$ & $100.0 \%$ \\
\hline $\mathrm{COU}$ & $75.9 \%$ & $89.6 \%$ & $80.2 \%$ & $23.9 \%$ & $9.9 \%$ & $19.4 \%$ & $0.1 \%$ & $0.5 \%$ & $0.2 \%$ & $0.1 \%$ & $0.1 \%$ & $0.1 \%$ & $0.0 \%$ & $0.0 \%$ & $0.0 \%$ & $100.0 \% \quad 100.0 \%$ & $100.0 \%$ \\
\hline TOTAL & $82.4 \%$ & $92.0 \%$ & $85.5 \%$ & $17.4 \%$ & $7.5 \%$ & $14.2 \%$ & $0.1 \%$ & $0.4 \%$ & $0.2 \%$ & $0.1 \%$ & $0.1 \%$ & $0.1 \%$ & $0.0 \%$ & $0.0 \%$ & $0.0 \%$ & $100.0 \% \quad 100.0 \%$ & $100.0 \%$ \\
\hline \multirow{2}{*}{ C: 90991} & \multicolumn{3}{|c|}{ INGLESS } & \multicolumn{3}{|c|}{ FRANCÉS } & \multicolumn{3}{|c|}{ ALEMÁN } & \multicolumn{3}{|c|}{ ITALIANO } & \multicolumn{3}{|c|}{ OTROS } & \multicolumn{2}{|l|}{ TOTAL } \\
\hline & Público & Privado & Total & Público & Privedo & Total & Público & Privado & Total & Público & Privado & Total & Público & Privado & Total & Público Privado & Total \\
\hline BUP 1 & $91.3 \%$ & $96.5 \%$ & $92.9 \%$ & $8.5 \%$ & $3.4 \%$ & $6.9 \%$ & $0.2 \%$ & $0.2 \%$ & $0.2 \%$ & $0.0 \%$ & $0.0 \%$ & $0.0 \%$ & $0.0 \%$ & & $0.0 \%$ & $100.0 \% \quad 100.0 \%$ & $100.0 \%$ \\
\hline BUP 2 & $87.8 \%$ & $95.5 \%$ & $90.3 \%$ & $12.0 \%$ & $4.3 \%$ & $9.5 \%$ & $0.1 \%$ & $0.2 \%$ & $0.2 \%$ & $0.0 \%$ & $0.0 \%$ & $0.0 \%$ & $0.0 \%$ & $0.0 \%$ & $0.0 \%$ & $100.0 \% \quad 100.0 \%$ & $100.0 \%$ \\
\hline BUP 3 & $84.0 \%$ & $94.1 \%$ & $87.4 \%$ & $15.8 \%$ & $5.8 \%$ & $12.4 \%$ & $0.2 \%$ & $0.2 \%$ & $0.2 \%$ & $0.0 \%$ & $0.0 \%$ & $0.0 \%$ & $0.0 \%$ & $0.0 \%$ & $0.0 \%$ & $100.0 \% \quad 100.0 \%$ & $100.0 \%$ \\
\hline $\mathrm{COU}$ & $80.6 \%$ & $92.8 \%$ & $84.4 \%$ & $19.3 \%$ & $7.0 \%$ & $15.4 \%$ & $0.1 \%$ & $0.1 \%$ & $0.1 \%$ & $0.0 \%$ & $0.0 \%$ & $0.0 \%$ & $0.0 \%$ & $0.0 \%$ & $0.0 \%$ & $100.0 \% \quad 100.0 \%$ & $100.0 \%$ \\
\hline TOTAL & $86.4 \%$ & $94.9 \%$ & $89.1 \%$ & $13.4 \%$ & $5.0 \%$ & $10.7 \%$ & $0.1 \%$ & $0.2 \%$ & $0.2 \%$ & $0.0 \%$ & $0.0 \%$ & $0.1 \%$ & $0.0 \%$ & $0.0 \%$ & $0.0 \%$ & $100.0 \% \quad 100.0 \%$ & $100.0 \%$ \\
\hline \multirow{2}{*}{ C: $91 / 92$} & \multicolumn{3}{|c|}{ INGLES } & \multicolumn{3}{|c|}{ FRANCÉS } & \multicolumn{3}{|c|}{ ALEMÁN } & \multicolumn{3}{|c|}{ ITALIANO } & \multicolumn{3}{|c|}{ OTROS } & \multicolumn{2}{|l|}{ TOTAL } \\
\hline & Público & Privado & Total & Público & Privado & Total & Público & Privado & Total & Público & Privado & Total & Público & Privado & Total & Público Privado & Total \\
\hline BUP 1 & $93.3 \%$ & $97.7 \%$ & $94.7 \%$ & $6.4 \%$ & $2.0 \%$ & $5.0 \%$ & $0.2 \%$ & $0.2 \%$ & $0.2 \%$ & $0.1 \%$ & $0.1 \%$ & $0.1 \%$ & $0.0 \%$ & 0.0 & $0.0 \%$ & $100.0 \% \quad 100.0 \%$ & $100.0 \%$ \\
\hline BUP 2 & $90.1 \%$ & $97.1 \%$ & $92.3 \%$ & $9.6 \%$ & $2.7 \%$ & $7.4 \%$ & $0.2 \%$ & $0.2 \%$ & $0.2 \%$ & $0.1 \%$ & $0.1 \%$ & $0.1 \%$ & $0.0 \%$ & $0.0 \%$ & $0.0 \%$ & $100.0 \% \quad 100.0 \%$ & $100.0 \%$ \\
\hline BUP 3 & $87.6 \%$ & $96.0 \%$ & $90.3 \%$ & $12.2 \%$ & $3.7 \%$ & $9.4 \%$ & $0.1 \%$ & $0.2 \%$ & $0.2 \%$ & $0.1 \%$ & $0.1 \%$ & $0.1 \%$ & $0.0 \%$ & $0.0 \%$ & $0.0 \%$ & $100.0 \% \quad 100.0 \%$ & $100.0 \%$ \\
\hline $\mathrm{COU}$ & $84.0 \%$ & $94.3 \%$ & $87.2 \%$ & $15.8 \%$ & $5.5 \%$ & $12.6 \%$ & $0.1 \%$ & $0.1 \%$ & $0.1 \%$ & $0.1 \%$ & $0.1 \%$ & 0.19 & $0.0 \%$ & $0.0 \%$ & $0.0 \%$ & $100.0 \% \quad 100.0 \%$ & $100.0 \%$ \\
\hline TOTAL & $89.0 \%$ & $96.4 \%$ & $91.4 \%$ & $10.7 \%$ & $3.3 \%$ & $8.4 \%$ & $0.2 \%$ & $0.2 \%$ & $0.2 \%$ & $0.1 \%$ & $0.1 \%$ & $0.1 \%$ & $0.0 \%$ & $0.0 \%$ & $0.0 \%$ & $100.0 \% \quad 100.0 \%$ & $100.0 \%$ \\
\hline
\end{tabular}




\begin{tabular}{|c|c|c|c|c|c|c|c|c|c|c|c|c|c|c|c|c|c|}
\hline \multicolumn{18}{|c|}{ MEC ALUMNOS DE B.U.P y C.O.U. que cursan idioma optativo } \\
\hline \multirow{2}{*}{ C: $89 / 90$} & \multicolumn{3}{|c|}{ INGLES } & \multicolumn{3}{|c|}{ FRANCÉS } & \multicolumn{3}{|c|}{ ALEMÁN } & \multicolumn{3}{|c|}{ ITALLANO } & \multicolumn{3}{|c|}{ OTROS } & \multicolumn{2}{|l|}{ TOTAL } \\
\hline & Público & Privado & Total & Público & Privado & Total & Público $F$ & Privado & Total & Püblico $\operatorname{Pr}$ & Privado & Total & Público & Privado & Total & Público Privado & Total \\
\hline BUP 1 & $9.1 \%$ & $21.4 \%$ & $11.6 \%$ & $87.7 \%$ & $77.5 \%$ & $85.7 \%$ & $2.1 \%$ & $1.1 \%$ & 1.99 & $1.0 \%$ & $0.0 \%$ & $0.8 \%$ & $0.0 \%$ & $0.0 \%$ & $0.0 \%$ & $100.0 \% 100.0 \%$ & $100.0 \%$ \\
\hline BUP 2 & $10.4 \%$ & $30.9 \%$ & $14.8 \%$ & $86.9 \%$ & $60.6 \%$ & $81.3 \%$ & $2.0 \%$ & $4.0 \%$ & $2.5 \%$ & $0.5 \%$ & $4.4 \%$ & $1.4 \%$ & $0.1 \%$ & $0.0 \%$ & $0.1 \%$ & $100.0 \% 100.0 \%$ & $100.0 \%$ \\
\hline BUP 3 & $14.0 \%$ & $40.1 \%$ & $21.5 \%$ & $81.5 \%$ & $51.5 \%$ & 72.99 & $1.8 \%$ & $2.9 \%$ & $2.1 \%$ & $2.6 \%$ & $5.6 \%$ & $3.4 \%$ & $0.1 \%$ & $0.0 \%$ & $0.1 \%$ & $100.0 \% \quad 100.0 \%$ & $100.0 \%$ \\
\hline TOTAL & $10.1 \%$ & $27.7 \%$ & $13.9 \%$ & $86.7 \%$ & $67.5 \%$ & $82.6 \%$ & $2.0 \%$ & $2.3 \%$ & $2.1 \%$ & $1.1 \%$ & $2.4 \%$ & $1.3 \%$ & $0.1 \%$ & $0.0 \%$ & $0.1 \%$ & $100.0 \% \quad 100.0 \%$ & $100.0 \%$ \\
\hline \multirow{2}{*}{ C: 90991} & \multicolumn{3}{|c|}{ INGLES } & \multicolumn{3}{|c|}{ FRANCÉS } & \multicolumn{3}{|c|}{ ALEMÁN } & \multicolumn{3}{|c|}{ ITALIANO } & \multicolumn{3}{|c|}{ OTROS } & \multicolumn{2}{|l|}{ TOTAL } \\
\hline & Público & Privado & Total & Público & Privado & Total & Público $P$ & Privado & Total & Püblico $P n$ & Privado & Total & Público & Privado & Total & Público Privado & Total \\
\hline BUP 1 & $5.3 \%$ & $21.5 \%$ & $8.7 \%$ & & $76.2 \%$ & $88.9 \%$ & $2.3 \%$ & $2.3 \%$ & $2.3 \%$ & $0.0 \%$ & $0.0 \%$ & $0.0 \%$ & $0.0 \%$ & $0.0 \%$ & $0.0 \%$ & $100.0 \% \quad 100.0 \%$ & $100.0 \%$ \\
\hline BUP 2 & $6.2 \%$ & $20.0 \%$ & $9.5 \%$ & $91.8 \%$ & $78.2 \%$ & $88.5 \%$ & $2.0 \%$ & $1.9 \%$ & $1.9 \%$ & $0.0 \%$ & $0.0 \%$ & $0.0 \%$ & $0.0 \%$ & $0.0 \%$ & $0.0 \%$ & $100.0 \% \quad 100.0 \%$ & $100.0 \%$ \\
\hline BUP 3 & $8.3 \%$ & $24.1 \%$ & $11.3 \%$ & $89.8 \%$ & $73.4 \%$ & $86.6 \%$ & $1.9 \%$ & $2.5 \%$ & $2.1 \%$ & $0.0 \%$ & $0.0 \%$ & $0.0 \%$ & $0.0 \%$ & $0.0 \%$ & $0.0 \%$ & $100.0 \% 1000 \%$ & $100.0 \%$ \\
\hline TOTAL & $6.2 \%$ & $21.4 \%$ & $9.4 \%$ & $91.7 \%$ & $76.4 \%$ & $87.4 \%$ & $2.1 \%$ & $2.2 \%$ & $2.1 \%$ & $0.0 \%$ & $0.0 \%$ & $0.7 \%$ & $0.0 \%$ & $0.0 \%$ & $0.4 \%$ & $100.0 \% 1000 \%$ & $100.0 \%$ \\
\hline \multirow{2}{*}{$C: 91 / 92$} & \multicolumn{3}{|c|}{ INGLÉS } & \multicolumn{3}{|c|}{ FRANCÉS } & \multicolumn{3}{|c|}{ ALEMÁN } & \multicolumn{3}{|c|}{ ITALIANO } & \multicolumn{3}{|c|}{ OTROS } & \multicolumn{2}{|l|}{ TOTAL } \\
\hline & Püblico & Privado & Total & Püblico & Privado & Total & Público $P$ & Privado & Total & Público $P r$ & Privado & Total & Público & Privado & Total & Público Privado & Total \\
\hline BUP 1 & $5.0 \%$ & $10.5 \%$ & $6.1 \%$ & $91.6 \%$ & $87.5 \%$ & $90.8 \%$ & $3.0 \%$ & $2.0 \%$ & $2.8 \%$ & $0.5 \%$ & $0.0 \%$ & $0.4 \%$ & $0.0 \%$ & $0.0 \%$ & $0.0 \%$ & $100.0 \% \quad 100.0 \%$ & $100.0 \%$ \\
\hline BUP 2 & $6.3 \%$ & $10.7 \%$ & $7.2 \%$ & $91.1 \%$ & $87.5 \%$ & $90.3 \%$ & $2.1 \%$ & $1.5 \%$ & $2.0 \%$ & $0.6 \%$ & $0.2 \%$ & $0.5 \%$ & $0.0 \%$ & $0.0 \%$ & $0.0 \%$ & $100.0 \% \quad 100.0 \%$ & $100.0 \%$ \\
\hline BUP 3 & $9.4 \%$ & $10.8 \%$ & $9.8 \%$ & $87.9 \%$ & $82.3 \%$ & $86.6 \%$ & $2.0 \%$ & $5.4 \%$ & $2.8 \%$ & $0.7 \%$ & $1.5 \%$ & $0.9 \%$ & $0.0 \%$ & $0.0 \%$ & $0.0 \%$ & $100.0 \% \quad 100.0 \%$ & $100.0 \%$ \\
\hline TOTAL & $6.4 \%$ & $10.6 \%$ & $7.3 \%$ & $90.6 \%$ & $86.2 \%$ & $89.6 \%$ & $2.4 \%$ & $2.7 \%$ & $2.5 \%$ & $0.6 \%$ & $0.4 \%$ & $0.5 \%$ & $0.0 \%$ & $0.0 \%$ & $0.0 \%$ & $100.0 \% \quad 100.0 \%$ & $100.0 \%$ \\
\hline
\end{tabular}




\begin{tabular}{|c|c|c|c|c|c|c|c|c|c|c|c|c|c|c|c|c|c|c|}
\hline \multicolumn{19}{|c|}{ ANEXO 2} \\
\hline \multicolumn{19}{|c|}{ ALUMNOS DE F.P. que cursan idioma obligatorio } \\
\hline \multirow{2}{*}{ C: 8990} & \multicolumn{3}{|c|}{ INGLÉS } & \multicolumn{3}{|c|}{ FRANCÉS } & \multicolumn{3}{|c|}{ ALEMÁN } & \multicolumn{3}{|c|}{ TIALANO } & \multicolumn{3}{|c|}{ OTROS } & \multicolumn{3}{|c|}{ TOTAL } \\
\hline & Público & Privado & Total & Público & Privado & Total & Püblico & Privado & Total & Público & Privado & Total & Público & Privado & Total & Público & Privado & Total \\
\hline 1 GRADO & 91230 & 50320 & 141550 & 14301 & 3038 & 17339 & 58 & 0 & 58 & & & 0 & & & 0 & 105589 & 53358 & 158947 \\
\hline 2GRADO & 72148 & 30313 & 102461 & 14737 & 1327 & 16064 & 57 & 55 & 112 & & & 0 & & & 0 & 86942 & 31695 & 118637 \\
\hline TOTAL & 163378 & 80633 & 244011 & 29038 & 4365 & 33403 & 115 & 55 & 170 & 0 & 0 & 0 & 0 & 0 & 0 & 192531 & 85053 & 277584 \\
\hline \multirow{2}{*}{ C: 90091} & \multicolumn{3}{|c|}{ INGLESS } & \multicolumn{3}{|c|}{ FRANCÉS } & \multicolumn{3}{|c|}{ ALEMÁN } & \multicolumn{3}{|c|}{ ITALANO } & \multicolumn{3}{|c|}{ OTROS } & \multicolumn{3}{|c|}{ TOTAL } \\
\hline & Público & Privado & Total & Público & Privado & Total & Público & Privado & Total & Público & Privado & Total & Público & Privado & Total & Püblico & Privado & Total \\
\hline 1 GRADD & 91381 & 48259 & 139640 & 11827 & 2426 & 14253 & 0 & 0 & 0 & & & 0 & & & 0 & 103208 & 50685 & 153893 \\
\hline ACCÉSO & 3022 & 763 & 3785 & 688 & 28 & 716 & 0 & 0 & 0 & & & 0 & & & 0 & 3710 & 791 & 4501 \\
\hline 2 GRADO & 75276 & 28213 & 103489 & 14007 & 1013 & 15020 & 33 & 0 & 33 & & & 0 & & & 0 & 89316 & 29226 & 118542 \\
\hline TOTAL & 169679 & 77235 & 246914 & 26522 & 3467 & 29989 & 33 & 0 & 33 & 0 & 0 & 0 & 0 & 0 & 0 & 196234 & 80702 & 276936 \\
\hline \multirow{2}{*}{ C: $91 / 92$} & \multicolumn{3}{|c|}{ INGLÉS } & \multicolumn{3}{|c|}{ FRANCÉS } & \multicolumn{3}{|c|}{ ALEMÁN } & \multicolumn{3}{|c|}{ ITALANO } & \multicolumn{3}{|c|}{ OTROS } & \multicolumn{3}{|c|}{ TOTAL } \\
\hline & Público & Privado & Total & Público & Privado & Total & Público & Privado & Total & Público & Privado & Total & Público & Privado & Total & Público & Privado & Total \\
\hline 1 GRADO & 89129 & 46730 & 135859 & 8820 & 1924 & 10744 & 112 & 0 & 112 & & & 0 & & & 0 & 98061 & 48654 & 146715 \\
\hline ACCÉSO & 3643 & 755 & 439 & 712 & 16 & 728 & 0 & 0 & 0 & & & 0 & & & 0 & 4355 & 5771 & 5126 \\
\hline 2 GRADO & 84614 & 26994 & 111600 & 13669 & 1026 & 14695 & 40 & 2 & 42 & & & 0 & & & 0 & 98323 & 328022 & 126345 \\
\hline TOTAL & 177386 & 74479 & 251865 & 23201 & 2966 & 26167 & 152 & 2 & 154 & 0 & 0 & 0 & 0 & 0 & 0 & 200739 & 77447 & 278186 \\
\hline
\end{tabular}




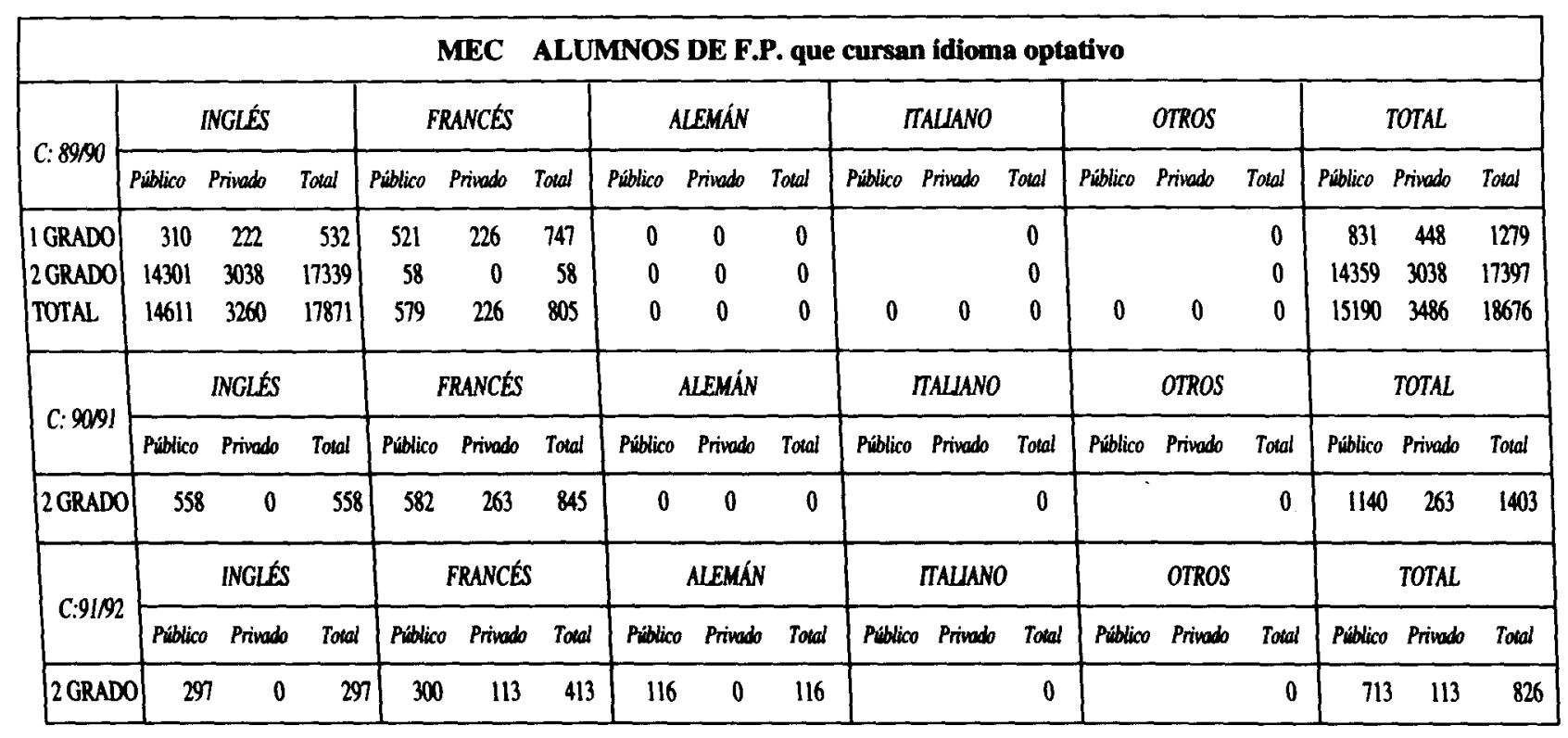

w 
ANEXo 2.1

\begin{tabular}{|c|c|c|c|c|c|c|c|c|c|c|c|c|c|c|c|c|c|}
\hline \multicolumn{18}{|c|}{ MEC ALUMNOS DE F.P. que cursan idioma obligatorio } \\
\hline \multirow{2}{*}{$C: 89 / 90$} & \multicolumn{3}{|c|}{ INGLÉS } & \multicolumn{3}{|c|}{ FRANCESS } & \multicolumn{3}{|c|}{ ALEMÁN } & \multicolumn{3}{|c|}{ ITALANO } & \multicolumn{3}{|c|}{ OTROS } & \multicolumn{2}{|l|}{ TOTAL } \\
\hline & Publico & Privado & Total & Püblico & Privedo & Total & Püblico & Privado & Total & Püblico & Privedo & Total & Piblico & Privado & Total & Público Privado & Total \\
\hline 1 GRADO & $86.4 \%$ & $94.3 \%$ & $89.1 \%$ & $13.5 \%$ & $5.7 \%$ & $10.9 \%$ & $0.1 \%$ & $0.0 \%$ & $0.0 \%$ & $0.0 \%$ & $0.0 \%$ & $0.0 \%$ & $0.0 \%$ & $0.0 \%$ & $0.0 \%$ & $100.0 \% \quad 100.0 \%$ & $100.0 \%$ \\
\hline 2GRADO & $83.0 \%$ & $95.6 \%$ & $86.4 \%$ & $17.0 \%$ & $4.2 \%$ & $13.5 \%$ & $0.1 \%$ & $0.2 \%$ & $0.1 \%$ & $0.0 \%$ & $0.0 \%$ & $0.0 \%$ & $0.0 \%$ & $0.0 \%$ & $0.0 \%$ & $100.0 \% \quad 100.0 \%$ & $100.0 \%$ \\
\hline TOTAL & $84.9 \%$ & $94.8 \%$ & $87.9 \%$ & $15.1 \%$ & $5.1 \%$ & $12.0 \%$ & $0.1 \%$ & $0.1 \%$ & $0.1 \%$ & $0.0 \%$ & $0.0 \%$ & $0.0 \%$ & $0.0 \%$ & $0.0 \%$ & $0.0 \%$ & $100.0 \% \quad 100.0 \%$ & $100.0 \%$ \\
\hline \multirow{2}{*}{ C: 9091} & \multicolumn{3}{|c|}{ INGLESS } & \multicolumn{3}{|c|}{ FRANCÉS } & \multicolumn{3}{|c|}{ ALEMÁN } & \multicolumn{3}{|c|}{ MALLANO } & \multicolumn{3}{|c|}{ OTROS } & \multicolumn{2}{|l|}{ TOTAL } \\
\hline & Público & Privado & Total & Püblico & Privado & Total & Público & Privado & Total & Püblico & Privado & Total & Público & Privado & Total & Público Privado & Total \\
\hline 1 GRADO & $88.5 \%$ & $95.2 \%$ & $90.7 \%$ & $11.5 \%$ & $4.8 \%$ & $9.3 \%$ & $0.0 \%$ & $0.0 \%$ & $0.0 \%$ & $0.0 \%$ & $0.0 \%$ & $0.0 \%$ & $0.0 \%$ & $0.0 \%$ & $0.0 \%$ & $100.0 \% \quad 100.0 \%$ & $100.0 \%$ \\
\hline ACCÉSO & $81.5 \%$ & $9.5 \%$ & $84.1 \%$ & $18.5 \%$ & $3.5 \%$ & $15.9 \%$ & $0.0 \%$ & $0.0 \%$ & $0.0 \%$ & $0.0 \%$ & $0.0 \%$ & $0.0 \%$ & $0.0 \%$ & $0.0 \%$ & $0.0 \%$ & $100.0 \% \quad 100.0 \%$ & $100.0 \%$ \\
\hline 2 GRADO & $84.3 \%$ & $9.5 \%$ & $87.3 \%$ & $15.7 \%$ & $3.5 \%$ & $12.7 \%$ & $0.0 \%$ & $0.0 \%$ & $0.0 \%$ & $0.0 \%$ & $0.0 \%$ & $0.0 \%$ & $0.0 \%$ & $0.0 \%$ & $0.0 \%$ & $100.0 \% \quad 100.0 \%$ & $100.0 \%$ \\
\hline TOTAL & $86.5 \%$ & $95.7 \%$ & $89.2 \%$ & $13.5 \%$ & $4.3 \%$ & $10.8 \%$ & $0.0 \%$ & $0.0 \%$ & $0.0 \%$ & $0.0 \%$ & $0.0 \%$ & $0.0 \%$ & $0.0 \%$ & $0.0 \%$ & $0.0 \%$ & $100.0 \% \quad 100.0 \%$ & $100.0 \%$ \\
\hline \multirow{2}{*}{$C: 91 / 92$} & \multicolumn{3}{|c|}{ INGLÉS } & \multicolumn{3}{|c|}{ FRANCÉS } & \multicolumn{3}{|c|}{ ALEMÁN } & \multicolumn{3}{|c|}{ ITALIANO } & \multicolumn{3}{|c|}{ OTROS } & \multicolumn{2}{|l|}{ TOTAL } \\
\hline & Público & Privado & Total & Püblico & Privado & Total & Público & Privado & Total & Püblico & Privado & Tocal & Público & Privado & Total & Público Privado & Total \\
\hline 1 GRAD0 & $90.9 \%$ & $9.0 \%$ & $92.6 \%$ & $9.0 \%$ & $4.0 \%$ & $7.3 \%$ & $0.1 \%$ & $0.0 \%$ & $0.1 \%$ & $0.0 \%$ & $0.0 \%$ & $0.0 \%$ & $0.0 \%$ & $0.0 \%$ & $0.0 \%$ & $100.0 \% \quad 100.0 \%$ & $100.0 \%$ \\
\hline ACCÉSO & $83.7 \%$ & $97.9 \%$ & $85.8 \%$ & $16.3 \%$ & $2.1 \%$ & $14.2 \%$ & $0.0 \%$ & $0.0 \%$ & $0.0 \%$ & $0.0 \%$ & $0.0 \%$ & $0.0 \%$ & $0.0 \%$ & $0.0 \%$ & $0.0 \%$ & $100.0 \% \quad 100.0 \%$ & $100.0 \%$ \\
\hline 2 GRADO & $86.1 \%$ & $9.3 \%$ & $88.3 \%$ & $13.9 \%$ & $3.7 \%$ & $11.6 \%$ & $0.0 \%$ & $0.0 \%$ & $0.0 \%$ & $0.0 \%$ & $0.0 \%$ & $0.0 \%$ & $0.0 \%$ & $0.0 \%$ & $0.0 \%$ & $100.0 \% \quad 100.0 \%$ & $100.0 \%$ \\
\hline TOTAL & $88.4 \%$ & $96.2 \%$ & $90.5 \%$ & $11.6 \%$ & $3.8 \%$ & $9.4 \%$ & $0.1 \%$ & $0.0 \%$ & $0.1 \%$ & 0.09 & $0.0 \%$ & $0.0 \%$ & $0.0 \%$ & $0.0 \%$ & $0.0 \%$ & $100.0 \% \quad 100.0 \%$ & $100.0 \%$ \\
\hline
\end{tabular}




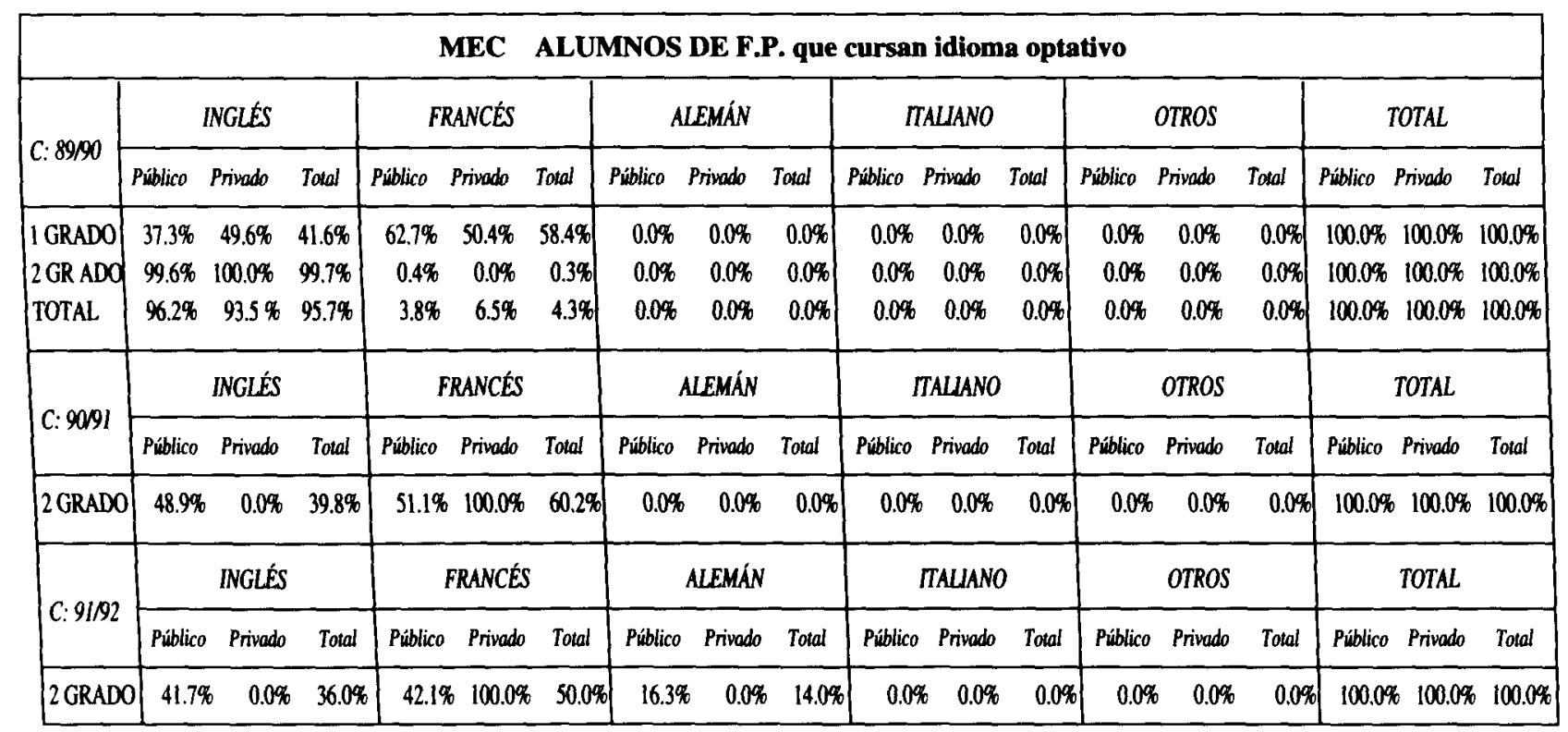

岕 
ANEXo 3

\begin{tabular}{|c|c|c|c|c|c|c|c|c|c|c|c|c|c|c|c|}
\hline \multicolumn{16}{|c|}{ ALUMNOS DE E.G.B. que cursan idiomas } \\
\hline \multirow{2}{*}{ C: 9091} & \multicolumn{3}{|c|}{ INGLESS } & \multicolumn{3}{|c|}{ FRANCES } & \multicolumn{3}{|c|}{ OTROS } & \multicolumn{3}{|c|}{$\begin{array}{c}\text { OTRAS LENGUAS } \\
\text { ESPA NOLLAS }\end{array}$} & \multicolumn{3}{|c|}{ TOTAL } \\
\hline & Priblico & Privado & Total & Público & Privado & Total & Público & Privado & Total & Público & Privado & Total & Público & Privado & Total \\
\hline TOTAL & 454091 & 297898 & 751989 & 55802 & 28659 & 84461 & 578 & 1975 & 2553 & 59911 & 41151 & 101062 & 570382 & 369883 & 940065 \\
\hline \multirow{2}{*}{$C: 9192$} & \multicolumn{3}{|c|}{ INGLES } & \multicolumn{3}{|c|}{ FRANCES } & \multicolumn{3}{|c|}{ OTROS } & \multicolumn{3}{|c|}{$\begin{array}{c}\text { OTRAS LENGUAS } \\
\text { ESPAN̂́OLAS }\end{array}$} & \multicolumn{3}{|c|}{ TOTAL } \\
\hline & Píblico & Privado & Total & Público & Privado & Toxal & Público & Privado & Total & Público & Privado & Total & Público & Privado & Total \\
\hline TOTAL & 525329 & 293941 & 819270 & 40194 & 26430 & 66624 & 648 & 1198 & 1846 & 59227 & 40532 & 99759 & 625398 & 362101 & 987499 \\
\hline
\end{tabular}


ANEXo 3.1

\begin{tabular}{|c|c|c|c|c|c|c|c|c|c|c|c|c|c|c|c|}
\hline \multicolumn{16}{|c|}{ MEC ALUMNOS DE E.G.B. que cursan idiomas } \\
\hline \multirow{2}{*}{ C: 9091} & \multicolumn{3}{|c|}{ INGLÉS } & \multicolumn{3}{|c|}{ FRANCÉS } & \multicolumn{3}{|c|}{ OTROS } & \multicolumn{3}{|c|}{$\begin{array}{l}\text { OTRAS LENGUAS } \\
\text { ESPAÑOLAS }\end{array}$} & \multicolumn{3}{|c|}{ TOTAL } \\
\hline & Público & Privado & Total & Público & Privado & Total & Público & Privado & Total & Público & Privado & Total & Público & Privado & Total \\
\hline TOTAL & $79.6 \%$ & $80.6 \%$ & $80.0 \%$ & $9.8 \%$ & $7.8 \%$ & $9.0 \%$ & $0.1 \%$ & $0.5 \%$ & $0.3 \%$ & $10.5 \%$ & $11.1 \%$ & $10.8 \%$ & $100.0 \%$ & $100.0 \%$ & $100.0 \%$ \\
\hline \multirow{2}{*}{$C: 91 / 92$} & \multicolumn{3}{|c|}{ INGLES } & \multicolumn{3}{|c|}{ FRANCÉS } & \multicolumn{3}{|c|}{ OTROS } & \multicolumn{3}{|c|}{$\begin{array}{l}\text { OTRAS LENGUAS } \\
\text { ESPAÑOLAS }\end{array}$} & \multicolumn{3}{|c|}{ TOTAL } \\
\hline & Priblico & Privado & Tocal & Público & Privado & Total & Público & Privado & Total & Público & Privado & Total & Público & Privado & Total \\
\hline TOTAL & $84.0 \%$ & $81.2 \%$ & $83.0 \%$ & $6.4 \%$ & $7.3 \%$ & $6.7 \%$ & $0.1 \%$ & $0.3 \%$ & $0.2 \%$ & $9.5 \%$ & $11.2 \%$ & $10.1 \%$ & $100.0 \%$ & $100.0 \%$ & $100.0 \%$ \\
\hline
\end{tabular}


ANEXo 4

\begin{tabular}{|c|c|c|c|c|c|c|c|c|c|c|c|c|c|c|c|}
\hline \multicolumn{16}{|c|}{ cursan idioma obligatorio } \\
\hline \multirow{2}{*}{ C: $89 / 90$} & \multicolumn{3}{|c|}{ INGLÉS } & \multicolumn{3}{|c|}{ FRANCÉS } & \multicolumn{3}{|c|}{ ALEMÁN } & \multicolumn{3}{|c|}{ OTROS } & \multicolumn{3}{|c|}{ TOTAL } \\
\hline & Público & Privado & Total & Püblico & Privado & Total & Público & Privado & Total & Público & Privado & Total & Público & Privado & Total \\
\hline $\mathrm{C} 1-1$ & 2102 & 301 & 2403 & 300 & 34 & 334 & 0 & 0 & 0 & 0 & 0 & 0 & 2402 & 335 & 2737 \\
\hline C.1-2 & 1539 & 262 & 1801 & 240 & 34 & 274 & 0 & 0 & 0 & 0 & 0 & 0 & 1779 & 296 & 2075 \\
\hline C.2-1 & 1220 & 207 & 1427 & 343 & 25 & 368 & 0 & 0 & 0 & 0 & 0 & 0 & 1563 & 232 & 1795 \\
\hline C. $2-2$ & 929 & 172 & 1101 & 341 & 42 & 383 & 0 & 0 & 0 & 0 & 0 & 0 & 1270 & 214 & 1484 \\
\hline TOTAL & 5790 & 942 & 6732 & 1224 & 135 & 1359 & 0 & 0 & 0 & 0 & 0 & 0 & 7014 & 1077 & 8091 \\
\hline \multirow{2}{*}{ C: 90091} & \multicolumn{3}{|c|}{ INGLES } & \multicolumn{3}{|c|}{ FRANCÉS } & \multicolumn{3}{|c|}{ ALEMÁN } & \multicolumn{3}{|c|}{ OTROS } & \multicolumn{3}{|c|}{ TOTAL } \\
\hline & Püblico & Privado & Toval & Público & Privado & Total & Público & Privado & Total & Público & Privado & Total & Público & Privado & Total \\
\hline$C 1-1$ & 12607 & 1065 & 13672 & 1788 & 36 & 1824 & 0 & 0 & 0 & 0 & 0 & 0 & 14395 & 1101 & $154 \%$ \\
\hline C. $1-2$ & 4660 & 857 & 5517 & 751 & 32 & 783 & 0 & 0 & 0 & 0 & 0 & 0 & 5411 & 889 & 6300 \\
\hline C.2-1 & 1998 & 433 & 2431 & 381 & 27 & 408 & 0 & 0 & 0 & 0 & 0 & 0 & 2379 & 460 & 2839 \\
\hline C. $2-2$ & 1784 & 381 & 2165 & 474 & 30 & 504 & 0 & 0 & 0 & 0 & 0 & 0 & 2258 & 411 & 2669 \\
\hline TOTAL & 21049 & 2736 & 23785 & 3394 & 125 & 3519 & 0 & 0 & 0 & 0 & 0 & 0 & 24443 & 2861 & 27304 \\
\hline \multirow{2}{*}{$C: 91 / 92$} & \multicolumn{3}{|c|}{ INGLÉS } & \multicolumn{3}{|c|}{ FRANCÉS } & \multicolumn{3}{|c|}{ ALEMÁN } & \multicolumn{3}{|c|}{ OTROS } & \multicolumn{3}{|c|}{ TOTAL } \\
\hline & Público & Privado & Total & Público & Privado & Total & Público & Privado & Total & Público & Privado & Total & Público & Privado & Total \\
\hline C $1-1$ & 25497 & 730 & 26227 & 2795 & 15 & 2810 & 0 & 0 & 0 & 0 & 0 & 0 & 28292 & 745 & 29037 \\
\hline C $1-2$ & 9189 & 774 & 9963 & 1261 & 18 & 1279 & 0 & 0 & 0 & 0 & 0 & 0 & 10450 & 792 & 11242 \\
\hline C 2-1 & 3359 & 423 & 3782 & 500 & 20 & 520 & 0 & 0 & 0 & 0 & 0 & 0 & 3859 & 443 & 4302 \\
\hline C $2-2$ & 1993 & 340 & 2333 & 399 & 16 & 415 & 0 & 0 & 0 & 0 & 0 & 0 & 2392 & 356 & 2748 \\
\hline TOTAL & 40038 & 2267 & 42305 & 4955 & 69 & 5024 & 0 & 0 & 0 & 0 & 0 & 0 & 44993 & 2336 & 47329 \\
\hline
\end{tabular}




\begin{tabular}{|c|c|c|c|c|c|c|c|c|c|c|c|c|c|c|c|}
\hline \multicolumn{16}{|c|}{ ALUMNOS DEL PLAN EXPERIMENTAL (REM) que cursan idioma optativo } \\
\hline \multirow{2}{*}{ C: 9091} & \multicolumn{3}{|c|}{ INGLES } & \multicolumn{3}{|c|}{ FRANCES } & \multicolumn{3}{|c|}{ ALEMÁN } & \multicolumn{3}{|c|}{ OTROS } & \multicolumn{3}{|c|}{ TOTAL } \\
\hline & Público & Privado & Total & Público & Privado & Total & Püblico & Privado & Total & Público & Privado & Total & Público & Privado & Total \\
\hline C.2 & 191 & 15 & 206 & 605 & 148 & 753 & 8 & 22 & 30 & 0 & 0 & 0 & 804 & 185 & 989 \\
\hline \multirow{2}{*}{$C: 91 / 92$} & \multicolumn{3}{|c|}{ INGLES } & \multicolumn{3}{|c|}{ FRANCÉS } & \multicolumn{3}{|c|}{ ALEMÁN } & \multicolumn{3}{|c|}{ OTROS } & \multicolumn{3}{|c|}{ TOTAL } \\
\hline & Público & Privado & Tolal & Público & Privado & Total & Público & Privado & Toual & Público & Privado & Total & Público & Privado & Total \\
\hline C. 2 & 344 & 33 & 377 & 914 & 46 & 960 & 42 & 0 & 42 & 26 & 0 & 26 & 1326 & 79 & 1405 \\
\hline
\end{tabular}


ANEXO 4.1

\begin{tabular}{|c|c|c|c|c|c|c|c|c|c|c|c|c|c|c|c|}
\hline \multicolumn{16}{|c|}{ EL PLAN EXPERIMENTAL (REM) que cursan idioma obligatorio } \\
\hline \multirow{2}{*}{$C: 89 / 90$} & \multicolumn{3}{|c|}{ INGLÉS } & \multicolumn{3}{|c|}{ FRANCÉS } & \multicolumn{3}{|c|}{ ALEMÁN } & \multicolumn{3}{|c|}{ OTROS } & \multicolumn{3}{|c|}{ TOTAL } \\
\hline & Público & Privado & Total & Público & Privado & Total & Público & Privado & Total & Público & Privado & Total & Público & Privado & Total \\
\hline C.1-1 & $87.5 \%$ & $89.9 \%$ & $87.8 \%$ & $12.5 \%$ & $10.1 \%$ & $12.2 \%$ & $0.0 \%$ & $0.0 \%$ & $0.0 \%$ & $0.0 \%$ & $0.0 \%$ & $0.0 \%$ & $100.0 \%$ & $100.0 \%$ & $100.0 \%$ \\
\hline C. 1.2 & $86.5 \%$ & $88.5 \%$ & $86.8 \%$ & $13.5 \%$ & $11.5 \%$ & $13.2 \%$ & $0.0 \%$ & $0.0 \%$ & $0.0 \%$ & $0.0 \%$ & $0.0 \%$ & $0.0 \%$ & $100.0 \%$ & $100.0 \%$ & $100.0 \%$ \\
\hline C.2-1 & $78.1 \%$ & $89.2 \%$ & $79.5 \%$ & $21.9 \%$ & $10.8 \%$ & $20.5 \%$ & $0.0 \%$ & $0.0 \%$ & $0.0 \%$ & $0.0 \%$ & $0.0 \%$ & $0.0 \%$ & $100.0 \%$ & $100.0 \%$ & $100.0 \%$ \\
\hline C.2-2 & $73.1 \%$ & $80.4 \%$ & $74.2 \%$ & $26.9 \%$ & $19.6 \%$ & $25.8 \%$ & $0.0 \%$ & $0.0 \%$ & $0.0 \%$ & $0.0 \%$ & $0.0 \%$ & $0.0 \%$ & $100.0 \%$ & $100.0 \%$ & $100.0 \%$ \\
\hline TOTAL & $82.5 \%$ & $87.5 \%$ & $83.2 \%$ & $17.5 \%$ & $12.5 \%$ & $16.8 \%$ & $0.0 \%$ & $0.0 \%$ & $0.0 \%$ & $0.0 \%$ & $0.0 \%$ & $0.0 \%$ & $100.0 \%$ & $100.0 \%$ & $100.0 \%$ \\
\hline \multirow{2}{*}{ C: 90091} & \multicolumn{3}{|c|}{ INGLES } & \multicolumn{3}{|c|}{ FRANCÉS } & \multicolumn{3}{|c|}{ ALEMÁN } & \multicolumn{3}{|c|}{ OTROS } & \multicolumn{3}{|c|}{ TOTAL } \\
\hline & Píblico & Privado & Total & Público & Privado & Total & Público & Privado & Total & Público & Privado & Total & Público & Privado & Total \\
\hline C.1.1 & $87.6 \%$ & $96.7 \%$ & $88.2 \%$ & $12.4 \%$ & $3.3 \%$ & $11.8 \%$ & $0.0 \%$ & $0.0 \%$ & $0.0 \%$ & $0.0 \%$ & $0.0 \%$ & $0.0 \%$ & $100.0 \%$ & $100.0 \%$ & $100.0 \%$ \\
\hline C. $1-2$ & $86.1 \%$ & $96.4 \%$ & $87.6 \%$ & $13.9 \%$ & $3.6 \%$ & $12.4 \%$ & $0.0 \%$ & $0.0 \%$ & $0.0 \%$ & $0.0 \%$ & $0.0 \%$ & $0.0 \%$ & $100.0 \%$ & $100.0 \%$ & $100.0 \%$ \\
\hline C. $2-1$ & $84.0 \%$ & $94.1 \%$ & $85.6 \%$ & $16.0 \%$ & $5.9 \%$ & $14.4 \%$ & $0.0 \%$ & $0.0 \%$ & $0.0 \%$ & $0.0 \%$ & $0.0 \%$ & $0.0 \%$ & $100.0 \%$ & $100.0 \%$ & $100.0 \%$ \\
\hline C. $2-2$ & $79.0 \%$ & $92.7 \%$ & $81.1 \%$ & $21.0 \%$ & $7.3 \%$ & $18.9 \%$ & $0.0 \%$ & $0.0 \%$ & $0.0 \%$ & $0.0 \%$ & $0.0 \%$ & $0.0 \%$ & $100.0 \%$ & $100.0 \%$ & $100.0 \%$ \\
\hline TOTAL & $86.1 \%$ & $95.6 \%$ & $87.1 \%$ & $13.9 \%$ & $4.4 \%$ & $12.9 \%$ & $0.0 \%$ & $0.0 \%$ & $0.0 \%$ & $0.0 \%$ & $0.0 \%$ & $0.0 \%$ & $100.0 \%$ & $100.0 \%$ & $100.0 \%$ \\
\hline \multirow{2}{*}{$C: 91 / 92$} & \multicolumn{3}{|c|}{ INGLÉS } & \multicolumn{3}{|c|}{ FRANCÉS } & \multicolumn{3}{|c|}{ ALEMÁN } & \multicolumn{3}{|c|}{ OTROS } & \multicolumn{3}{|c|}{ TOTAL } \\
\hline & Público & Privado & Total & Público & Privado & Total & Público & Privado & Total & Público & Privado & Total & Público & Privado & Total \\
\hline C.1-1 & $90.1 \%$ & $98.0 \%$ & $90.3 \%$ & $9.9 \%$ & $2.0 \%$ & $9.7 \%$ & $0.0 \%$ & $0.0 \%$ & $0.0 \%$ & $0.0 \%$ & $0.0 \%$ & $0.0 \%$ & $100.0 \%$ & $100.0 \%$ & $100.0 \%$ \\
\hline C. $1 \cdot 2$ & $87.9 \%$ & $97.7 \%$ & $88.6 \%$ & $12.1 \%$ & $2.3 \%$ & $11.4 \%$ & $0.0 \%$ & $0.0 \%$ & $0.0 \%$ & $0.0 \%$ & $0.0 \%$ & $0.0 \%$ & $100.0 \%$ & $100.0 \%$ & $100.0 \%$ \\
\hline C.2-1 & $87.0 \%$ & $95.5 \%$ & $87.9 \%$ & $13.0 \%$ & $4.5 \%$ & $12.1 \%$ & $0.0 \%$ & $0.0 \%$ & $0.0 \%$ & $0.0 \%$ & $0.0 \%$ & $0.0 \%$ & $100.0 \%$ & $100.0 \%$ & $100.0 \%$ \\
\hline C. $2-2$ & $83.3 \%$ & $95.5 \%$ & $84.9 \%$ & $16.7 \%$ & $4.5 \%$ & $15.1 \%$ & $0.0 \%$ & $0.0 \%$ & $0.0 \%$ & $0.0 \%$ & $0.0 \%$ & $0.0 \%$ & $100.0 \%$ & $100.0 \%$ & $100.0 \%$ \\
\hline TOTAL & $89.0 \%$ & $97.0 \%$ & $89.4 \%$ & $11.0 \%$ & $3.0 \%$ & $10.6 \%$ & $0.0 \%$ & $0.0 \%$ & $0.0 \%$ & $0.0 \%$ & $0.0 \%$ & $0.0 \%$ & $100.0 \%$ & $100.0 \%$ & $100.0 \%$ \\
\hline
\end{tabular}




\begin{tabular}{|c|c|c|c|c|c|c|c|c|c|c|c|c|c|c|c|}
\hline \multicolumn{16}{|c|}{ ALUMNOS DEL PLAN EXPERIMENTAL (REM) que cursan idioma optativo } \\
\hline \multirow{2}{*}{$C: 9091$} & \multicolumn{3}{|c|}{ NGLLES } & \multicolumn{3}{|c|}{ FRANCÉS } & \multicolumn{3}{|c|}{ ALEMÁN } & \multicolumn{3}{|c|}{ OTROS } & \multicolumn{3}{|c|}{ TOTAL } \\
\hline & Público & Privado & Total & Público & Privado & Total & Píblico & Privado & Total & Público & Privado & Total & Público & Privado & Total \\
\hline C.2 & $23.8 \%$ & $8.1 \%$ & $20.8 \%$ & 75.28 & $80.0 \%$ & $76.1 \%$ & $1.0 \%$ & $11.9 \%$ & $3.0 \%$ & $0.0 \%$ & $0.0 \%$ & $0.0 \%$ & $100.0 \%$ & $100.0 \%$ & $100.0 \%$ \\
\hline \multirow{2}{*}{$C: 91 / 92$} & \multicolumn{3}{|c|}{ INGLES } & \multicolumn{3}{|c|}{ FRANCES } & \multicolumn{3}{|c|}{ ALEMÁN } & \multicolumn{3}{|c|}{ OTROS } & \multicolumn{3}{|c|}{ TOTAL } \\
\hline & Público & Privado & Total & Público & Privado & Total & Público & Privado & Total & Público & Privado & Total & Público & Privado & Total \\
\hline C. 2 & $25.9 \%$ & $41.8 \%$ & $26.8 \%$ & $68.9 \%$ & 58.28 & $68.3 \%$ & $3.2 \%$ & $0.0 \%$ & $3.0 \%$ & $2.0 \%$ & $0.0 \%$ & $1.9 \%$ & $100.0 \%$ & $100.0 \%$ & $100.0 \%$ \\
\hline
\end{tabular}


ANEXo 5

\begin{tabular}{|c|c|c|c|}
\hline MEC & \multicolumn{3}{|c|}{ ALUMNOS DE B.U.P. que cursan idioma obligatorio } \\
\hline C: 9293 & INGLES & FRANCES & TOTAL \\
\hline B.U.P. & $92.07 \%$ & $7.93 \%$ & $100.00 \%$ \\
\hline \multicolumn{4}{|c|}{ MEC ALUMNOS DE B.U.P. que cursen idiona optativo } \\
\hline$C: 9293$ & INGLÉS & FRANCES & TOTAL \\
\hline B.U.P. & $3.32 \%$ & $96.68 \%$ & $100.00 \%$ \\
\hline \multicolumn{4}{|c|}{ MEC ALUMNOS DE F.P. que cursen idiom obligatorio } \\
\hline C: 92993 & INGLÉS & FRANCÉS & TOTAL \\
\hline B.U.P. & $90.00 \%$ & $10.00 \%$ & $100.00 \%$ \\
\hline \multicolumn{4}{|c|}{ MEC ALUMNOS DE E.G.B. que cursen idioma } \\
\hline C: 9293 & INGLESS & FRANCÉS & TOTAL \\
\hline B.U.P. & $94.91 \%$ & $5.09 \%$ & $100.00 \%$ \\
\hline
\end{tabular}

